\title{
Whole-body simultaneous time-of-flight PET-MRI: early experience with clinical studies
}

\author{
Ryogo Minamimoto ${ }^{*}$ Andrei lagaru, Mehran Jamali, Amir Barkodhodari, Dawn Holley, Shreyas Vasanawala, \\ Greg Zaharchuk
}

From PSMR 2015: 4th Conference on PET/MR and SPECT/MR

La Biodola, Isola d'Elba, Italy. 17-21 May 2015

Stanford University, Department of Radiology, Division of Nuclear Medicine and Molecular Imaging, USA
Recently, a whole-body, simultaneous positron emission tomography - magnetic resonance imaging (PET-MRI) system combing MRI with time-of-flight (TOF) PET has been developed. We present our initial experience with human clinical studies using 18Ffluorodeoxyglucase (FDG) with this scanner, in comparison to PET-CT. All patients underwent a single-injection of 18F-FDG, with a dual-imaging protocol consisting of PET-CT followed by PET-MRI scan. PET-MR attenuation correction used a two point Dixon fat-water separated method for the body, combined with registration to an atlas for the head. Two radiologists evaluated MRI image quality using the following scale (0 non-diagnostic; 1 poor; 2 good; 3 excellent). PET-MRI and PET-CT images were reviewed for FDG uptake thought to be consistent with malignancy by two readers independently, and categorized into 5 groups (1 both PETMRI and PET-CT positive, 2 PET-MRI positive, PET-CT positive in retrospect; 3 PET-CT positive, PET-MRI positive in retrospect; 4: PET-MRI positive, PET-CT negative; 5: PET-MRI negative, PET-CT positive) by consensus.

\section{Results}

Twenty-six oncologic patients (average age: $63 \pm 14$ yrs) with were enrolled in the study. PET-CT and PETMRI scan started $71 \pm 14$ and $144 \pm 22$ minutes after injection of $10 \AA 3$ mCi FDG, respectively. The average length of the PET-CT and PET-MRI scan was $20 \pm 7$, and $55 \pm 15$ minutes, respectively. All MRI images were rated to be diagnostic; $64 \%$ were rated excellent, $32 \%$ were rated good, and $4 \%$ were rated poor. $64 \%(34 / 53)$ of FDG intense lesions were observed in the same location for both PET-CT and PETMRI. TOF PET-MRI provided comparable image quality and diagnostic ability with PET-CT, despite imaging at a later time point.

Published: 18 May 2015

doi:10.1186/2197-7364-2-S1-A64

Cite this article as: Minamimoto et al:: Whole-body simultaneous time-of-flight PET-MRI: early experience with clinical studies. EJNMMI Physics 2015 2(Suppl 1):A64.

\section{SpringerOpen $^{\circ}$}

(c) 2015 Minamimoto et al; licensee Springer. This is an Open Access article distributed under the terms of the Creative Commons Attribution License (http://creativecommons.org/licenses/by/4.0), which permits unrestricted use, distribution, and reproduction in any medium, provided the original work is properly cited. 